\title{
Measurement of the quality factor of a new low- frequency differential accelerometer for testing the equivalence principle
}

\section{Citation}

lafolla, V., C. Lefevre, E. Fiorenza, F. Santoli, S. Nozzoli, C. Magnafico, M. Lucente, et al. 2014. "Measurement of the Quality Factor of a New Low-Frequency Differential Accelerometer for Testing the Equivalence Principle." Review of Scientific Instruments 85 (1) (January): 014502. doi:10.1063/1.4861349. http://dx.doi.org/10.1063/1.4861349.

\section{Published Version}

doi:10.1063/1.4861349

\section{Permanent link}

http://nrs.harvard.edu/urn-3:HUL.InstRepos:11857776

\section{Terms of Use}

This article was downloaded from Harvard University's DASH repository, and is made available under the terms and conditions applicable to Other Posted Material, as set forth at http:// nrs.harvard.edu/urn-3:HUL.InstRepos:dash.current.terms-of-use\#LAA

\section{Share Your Story}

The Harvard community has made this article openly available.

Please share how this access benefits you. Submit a story. 


\title{
Measurement of the quality factor of a new low-frequency differential accelerometer for testing the equivalence principle
}

\author{
V. Iafolla, ${ }^{1}$ C. Lefevre,${ }^{1}$ E. Fiorenza, ${ }^{1}$ F. Santoli, ${ }^{1}$ S. Nozzoli, ${ }^{1}$ C. Magnafico,${ }^{1}$ M. Lucente,${ }^{1}$ \\ D. Lucchesi, ${ }^{1}$ R. Peron, ${ }^{1}$ I. I. Shapiro, ${ }^{2}$ S. Glashow, ${ }^{3}$ and E. C. Lorenzini ${ }^{4}$ \\ ${ }^{1}$ Istituto di Astrofisica e Planetologia Spaziali (IAPS) and Istituto Nazionale di Astrofisica (INAF), \\ Via del Fosso del Cavaliere 100, 00133 Roma, Italy \\ ${ }^{2}$ Harvard-Smithsonian Center for Astrophysics (CfA), Cambridge, Massachusetts 02138, USA \\ ${ }^{3}$ Physics Department, Boston University, Boston, Massachusetts 02215, USA \\ ${ }^{4}$ Dipartimento di Ingegneria Industriale, Universita' degli Studi di Padova, Padova, Italy
}

(Received 29 May 2013; accepted 21 December 2013; published online 21 January 2014)

\begin{abstract}
A cryogenic differential accelerometer has been developed to test the weak equivalence principle to a few parts in $10^{15}$ within the framework of the general relativity accuracy test in an Einstein elevator experiment. The prototype sensor was designed to identify, address, and solve the major issues associated with various aspects of the experiment. This paper illustrates the measurements conducted on this prototype sensor to attain a high quality factor $\left(\mathrm{Q} \sim 10^{5}\right)$ at low frequencies $(<20 \mathrm{~Hz})$. Such a value is necessary for reducing the Brownian noise to match the target acceleration noise of $10^{-14} \mathrm{~g} / \sqrt{ } \mathrm{Hz}$, hence providing the desired experimental accuracy. () 2014 AIP Publishing LLC. [http://dx.doi.org/10.1063/1.4861349]
\end{abstract}

\section{INTRODUCTION}

GReAT (General Relativity Accuracy Test in an Einstein Elevator) is an experimental test to be carried out by the Experimental Gravitation Group (EGG) of IAPS (Institute for Space Astrophysics and Planetology) at INAF (National Institute for Astrophysics), aimed at verifying the Weak Equivalence Principle (WEP) with improved accuracy with respect to the state of the art through a balloon-based experiment. It is based on a detector housed in a balloon-borne capsule flying at an altitude of 40-45 km (Iafolla et al., ${ }^{1}$ Shapiro et al., ${ }^{2}$ and Lorenzini et al. ${ }^{3}$ ). After reaching the operating height, the capsule is released followed by the release of the detector inside the capsule, thus obtaining undisturbed free-fall conditions.

The core of the experiment is a new differential accelerometer that could enable the verification of the validity of the WEP with an accuracy of a few parts in $10^{15}$. Such a measurement would provide an improvement of 100 folds with respect to the current best results obtained with torsion balances (Schlamminger $e t a l .{ }^{4}$ ) and Lunar Laser Ranging (LLR) measurements (Williams et al. ${ }^{5}$ ) in the field of the Earth and of the Sun, respectively. Details about the experiment are provided in Iafolla et al. ${ }^{1,6,7}$

The Einstein Equivalence Principle (EEP) constitutes the basis of the general theory of relativity by Einstein and, in general, of other metric gravitational theories. So far physicists have formulated different versions of the principle (see Will ${ }^{9}$ and Nobili et al. ${ }^{10,11}$ for a summary). Among others, two main formulations can be identified: the WEP and the EEP.

The EEP states that results of any local non-gravitational experiment will be the same for an accelerated observer in free space and for a non-accelerated observer in a perfectly uniform gravitational field. The WEP is a weaker formulation of the EEP in which the statement is restricted just to the laws of motion of masses. These statements are the more-recent evolution of previous formulations.

Galilei's original formulation says that a free fall test mass (i.e., a body small enough to neglect the tidal gravitational forces and without other perturbing forces) follows a trajectory independent of its structure and composition. Equivalently, two different bodies placed in a gravitational field fall with the same acceleration-this statement is often named as the Universality of Free Fall (UFF). Galileo was the first to test the UFF, using two pendula of different composition to an accuracy of about $10^{-3}$ (Galilei, ${ }^{12}$ Fuligni and Iafolla, ${ }^{13}$ and Bramanti et al. ${ }^{14}$ ).

Newton's formulation states that the inertial mass defined by the 2nd law of dynamics is proportional to the gravitational mass defined by the gravitational law. By expressing the phenomenon through the second law of dynamics for inertial masses $\left(\mathrm{m}_{\mathrm{i}}\right), F=m_{i} a$, placed in a gravitational field, yields $m_{i} a=m_{g} g$, i.e., the UFF validity implies a direct proportionality between inertial mass and gravitational mass.

Finally, in 1907 Einstein provided a more comprehensive formulation: there is a "physical equivalence" between a gravitational field and an accelerated reference frame. Specifically, in a free fall system all masses fall in the same manner, hence the gravitational acceleration has no local dynamical effects (Nobili et al. ${ }^{10}$ ). All these statements express in different ways the so-called weak equivalence principle.

Any violation of the WEP would exhibit a differential acceleration between such objects when falling in a gravitational field. For this reason, the detector employed in the experiment GReAT is based on a differential accelerometer formed by two proof-masses of different materials.

Basically, testing the WEP means verifying the UFF through the measurement of the rate of fall of two test masses made of different materials in a gravitational field. Any tiny differential acceleration between them would indicate a WEP violation once all disturbing effects have been properly 
modeled or limited below the level of the differential accelerometer accuracy.

This paper describes some of the experimental activities carried out by the IAPS EGG to develop and test a new sensor within the framework of the GReAT experiment. The work reported hereafter deals with tests and measurements conducted on a prototype sensor designed to achieve a high quality factor (Q) at low frequency and cryogenic temperatures. Further details on the sensor, its design and developments are provided in Iafolla et al. ${ }^{7}$

From the scientific literature (Borrielli et al. ${ }^{15}$ and Serra et al. ${ }^{16}$ ), it is known experimentally that the $Q$ of high frequency oscillators increases as the temperature decreases. However, there is no experimental proof of a similar behavior for low frequency oscillators. The aim of this paper is to prove, through experimental results, that low frequency oscillators have the same behavior. At the same time, the measurement of the quality factor carried out on the new sensor proved that the values obtained are compatible with the accuracy required for the GReAT experiment. The prototype sensor cooled at cryogenic temperatures $(\sim 12 \mathrm{~K})$ achieved a $Q$ $\sim 1.5 \times 10^{5}$, thus keeping the Brownian noise at a level compatible with the accuracy goal of the free-fall experiment.

The paper is organized as follows: Section II gives a general description of the newly developed accelerometer and focuses on issues related to its quality factor; Sec. III illustrates in details the experimental tests conducted on the sensor and the associated results; and finally conclusions are drawn in Sec. IV.

\section{DIFFERENTIAL ACCELEROMETER AND QUALITY FACTOR}

Experiments designed for testing the WEP basically need two bodies of different composition free falling in a gravitational field and the ability to measure accurately their differential acceleration. Different ways are known to realize such a measurement: experiments can be carried out on-ground, in space, or at stratospheric altitudes, each one having advantages and disadvantages (Nobili ${ }^{11}$ ). GReAT belongs to the last type, utilizing a very sensitive differential accelerometer released in free fall inside a co-moving capsule previously released from an altitude of about $40 \mathrm{~km}$ (Iafolla et al. ${ }^{1}$ ). The physical observable is the Eötvös ratio $\eta$, defined as the ratio of the differential acceleration of the two falling masses $\left(a_{1}\right.$, $a_{2}$ ) to their average acceleration:

$$
\eta=2 \cdot \frac{a_{1}-a_{2}}{a_{1}+a_{2}} .
$$

A value of the Eötvös ratio different from zero would confirm a violation of the UFF and hence of the WEP. GReAT is based on a "null-measurement," i.e., if the equivalence principle is verified, both bodies will have the same behavior with respect to gravity and $\eta=O$ (within the accuracy of the measurement method). Alternatively, a differential acceleration detected above the noise level will result in $\eta \neq 0$, implying a violation of the WEP.

Differential accelerometers behave like mechanical oscillators and are consequently modeled as (very sensitive) har- monic oscillators. The detection of very small accelerations $a$ implies the measurement of small displacements $x$ which requires decreasing the oscillation frequency $(\nu=\omega / 2 \pi)$, as readily derived from $a=-\omega^{2} x$. Consequently, the sensor needs to operate at a relatively low frequency, of a few tens of $\mathrm{Hz}$, to achieve the required sensitivity.

Various noise sources contribute to masking the (possible) tiny differential signal detected by the sensor. Some of those sources are abated by the common mode rejection factor. Specifically, common disturbances stemming from the environment can be eliminated by either differencing the two output signals of the differential accelerometer or directly, in case of an intrinsically differential accelerometer design. Brownian noise also affects the signal but it cannot be removed through signal differencing: a high quality factor is needed to reduce its effect. Typically, considering a system with given mechanical characteristics, the quality factor can be increased by operating the sensor at cryogenic temperature, hence reducing the Brownian noise. However, this technique involves some challenges that need to be overcome.

After the last studies on GReAT (Iafolla et al. ${ }^{1,7}$ and Lorenzini et al. ${ }^{8}$ ), new advancements have been recently achieved through the design and manufacturing of new sensors (Iafolla et al. ${ }^{7}$ ). Several tests were carried out to address and solve key issues related to the experiment. Among them, a special attention was paid to the realization of a sensor with a quality factor high enough to reduce the Brownian noise to the level needed for the GReAT experiment. To achieve such a goal, different accelerometers were developed focusing the attention on the geometry of the mass suspensions that constitute the "springs" of the oscillators. On the basis of these studies and simulations, the EGG of IAPS has designed and constructed a new differential accelerometer based on the experience gained through the past laboratory experiments.

Figure 1 shows an exploded view of the new sensor formed by two intrinsically differential accelerometers joined together through a common frame.

Each single accelerometer consists of a test mass supported by two torsional suspensions with a special shape as shown later on. The mass is symmetrical with respect to the suspensions and accurately balanced in order to keep the oscillator at a stable equilibrium when subject to the gravitational field. In this way, mass elements are insensitive to linear accelerations and sensitive to angular accelerations. For this reason the accelerometer is called auto-differential. Moreover, angular accelerations produce equal effects on both oscillators and can be eliminated through differencing the outputs of the inner and outer proof masses.

The proof masses are suspended through their geometrical centers by means of two pairs of blades with cross-shaped section. The blades are the "spring" of the mechanical oscillator.

Each sensing mass (inner and outer) is sandwiched between two pairs of plates (one for each wing of the mass) to form a capacitive accelerometer (Fig. 1). Plates on one wing constitute the pick-up plates and provide sensing of any distance change with respect to the related proof mass. Plates on the opposite wing constitute the actuator/control plates that apply electrostatic forces to the sensing mass. The 


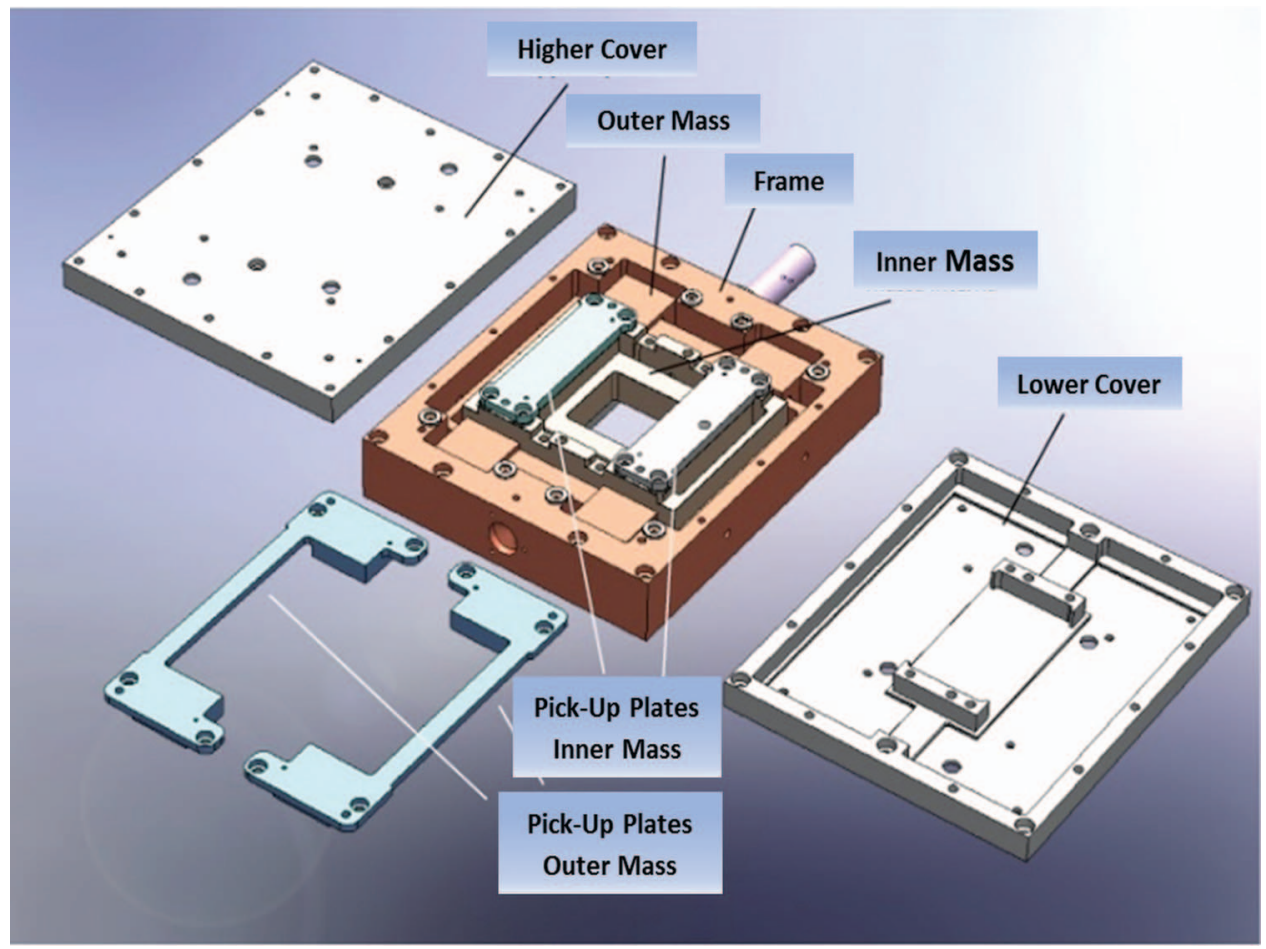

FIG. 1. Exploded view of the double auto-differential accelerometer designed by the EGG at IAPS.

displacement (i.e., rotation) of each single proof-mass from the equilibrium position is detected by means of the pick-up system based on capacitive transducers arranged in a bridgelike configuration.

The flight sensor is equipped with a system capable of rotating it around one of its main axes of inertia. Moments of inertia of the proof masses are the same by design in order to minimize gravity-gradient torques and precessional motions when the sensor is in free fall (Iafolla et al. ${ }^{6,7}$ ). Although the sensing masses have ideally the same moments of inertia, to reduce any spurious difference due to manufacturing errors and discriminate, through frequency separation, the movements of the free-falling system with respect to the useful signal, the blades have the torsional axis parallel to the sensor rotation axis (Shapiro et al. ${ }^{2}$ ).

The suspensions were designed for decoupling the torsional frequencies from flexural and longitudinal frequencies. Finite Element Method (FEM) simulations confirmed that a blade with cross-shaped section is best suited to provide the decoupling (Fig. 2).

The configuration adopted maximizes displacements when the proof masses are perturbed by torsional modes and minimizes displacements associated with bending modes. Consequently, the sensor has different torsional and flexural frequencies while those frequencies are identical for typical differential accelerometers. The cross-like shape of the blade, allowing an optimal decoupling between torsional and flexural modes, provides a low frequency for the torsion mode hence high sensitivity - and at the same time a high frequency for the flexural mode, yielding good structural stiffness. The final result is the realization of an accelerometer characterized by high sensitivity and good structural robustness.
Moreover, this new sensor geometry leads to a high rejection factor that produces a strong abatement of the differential noise. The configuration also leads to high values of the quality factor because the rotation axis of the sensitive masses crosses exactly the geometrical center of the torsional blades.

Figures 3 and 4 show the sensor core as manufactured by the milling machine at the IAPS laboratory.

Our EGG group carried out several tests on the differential accelerometer focusing on the measurement of the quality factor both at ambient and cryogenic temperatures.

For a general oscillating system, the quality factor is defined as the ratio of the energy stored in the system to the average energy dissipated per cycle (Jackson ${ }^{17}$ and

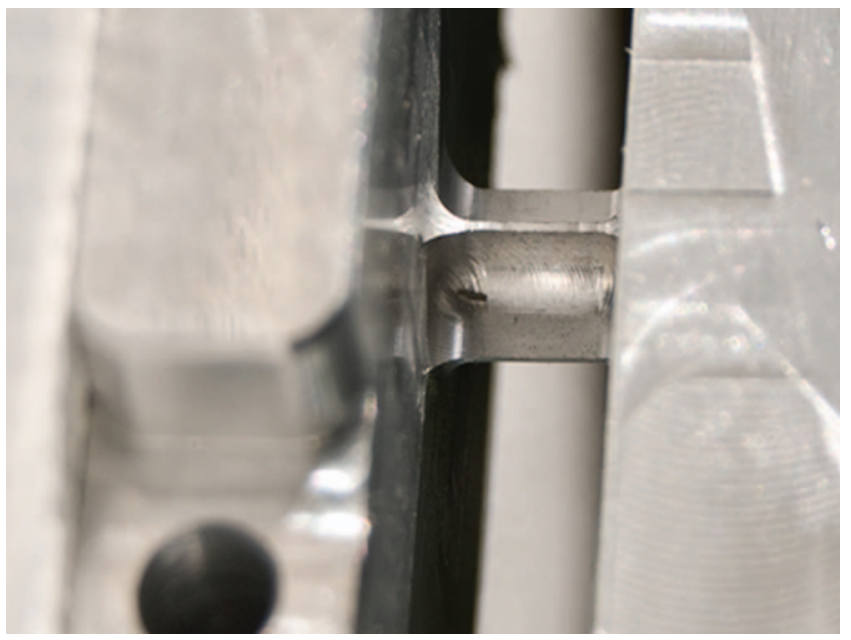

FIG. 2. Detail of the manufactured $0.4 \mathrm{~mm}$ cross-shaped blade. 


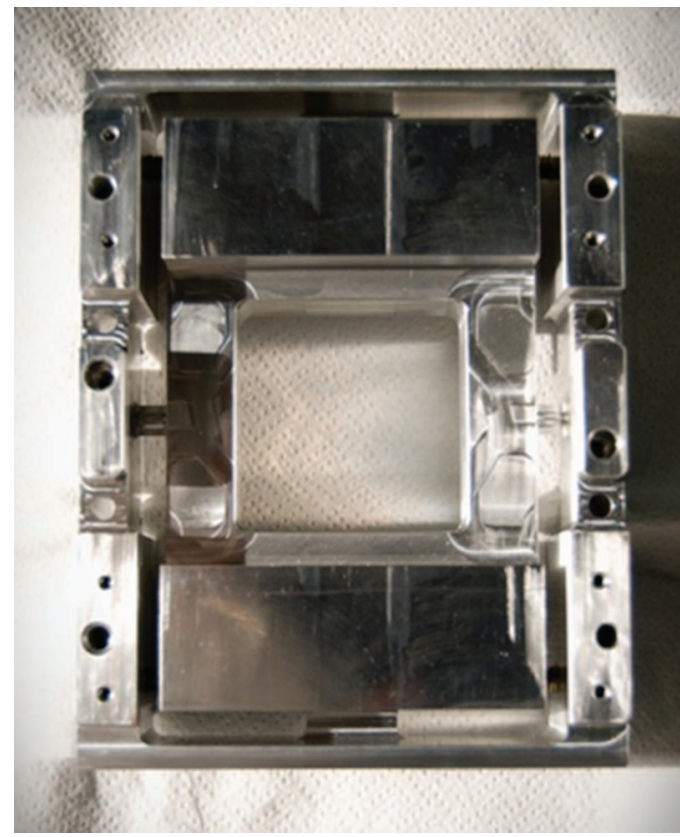

FIG. 3. Inner sensing mass at the end of the manufacturing process by the IAPS milling machine.

Hopcroft et al. $\left.{ }^{18}\right)$ :

$$
Q=2 \pi \frac{E_{\text {stored }}}{E_{\text {dissipated } / \text { cycle }}} .
$$

For small damping, the Q-factor is the ratio of the time constant $\tau$ of the amplitude decay of the oscillator to its oscillation period $\Omega(\mathrm{Q} \approx 2 \pi \tau / \Omega)$ : the higher the $\mathrm{Q}$ the lower the energy dissipation.

Based on the error budget of the GReAT experiment (see Table I from Iafolla et al. ${ }^{1}$ ), the achievement of the target accuracy of a few parts in $10^{15}$ requires a Brownian noise level below $10^{-14} \mathrm{~g} / \sqrt{ } \mathrm{Hz}$.

The Brownian mechanical noise can be translated into an equivalent acceleration (Gabrielson et al. ${ }^{19}$ ), yielding

$$
a_{B N}=\sqrt{\frac{4 k_{B} T 2 \pi v_{0}}{m Q}}\left[\frac{\mathrm{m} / \mathrm{s}^{2}}{\sqrt{H z}}\right],
$$

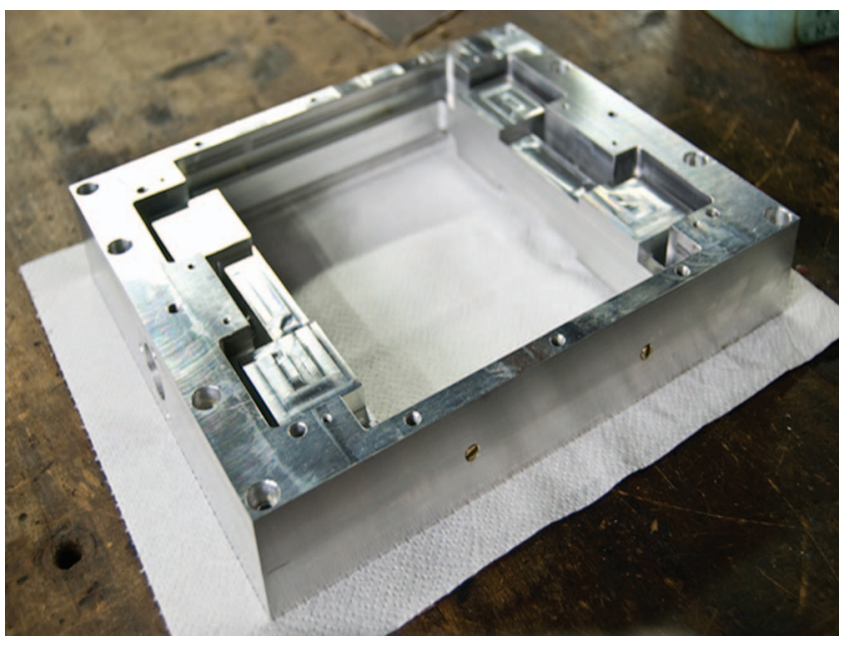

FIG. 4. Outer sensing mass after manufacturing.

where $m$ is the sensor mass, $v_{0}$ the mechanical resonance frequency of the differential mode, $Q$ the quality factor, $T$ the temperature, and $k_{B}$ the Boltzmann's constant.

Once the sensor parameters are defined at room temperature, the Brownian noise contribution can be reduced to a level consistent with the experimental accuracy by decreasing $T$ to cryogenic values, thus increasing the temperature-dependent $Q$. Assuming $T=4.2 \mathrm{~K}, v_{0}=10 \mathrm{~Hz}, m=10 \mathrm{~kg}, k_{B}=1.38$ $\times 10^{-23} \mathrm{~J} / \mathrm{K}$, and a Brownian noise acceleration target value $a_{B N}=10^{-14} \mathrm{~g} / \sqrt{ } \mathrm{Hz}$, Eq. (3) yields $Q \sim 1.5 \times 10^{5}$. Consequently, a $Q$ of order $10^{5}$ is required, among others, for achieving the stated performance of a few parts in $10^{15}$ in the free-fall experiment.

\section{MEASUREMENT OF THE QUALITY FACTOR}

A test campaign was carried out on different sensors both at ambient and cryogenic temperatures aimed at achieving the required sensitivity for GReAT. Among those, tests were made to verify the behavior of the quality factor versus temperature at low frequencies.

The experimental set-up included a cryogenic facility expressly developed to reach cryogenic temperatures down to 4.2 K. Figure 5 shows a drawing of the experiment within the cryogenic facility. The cryogenic facility is composed of

TABLE I. Error budget for the GReAT experiment (Iafolla et al. ${ }^{1}$ ). Symbol: $\mathrm{t}_{\mathrm{FALL}}=$ free-fall time, $\mathrm{t}_{\mathrm{int}}=$ integration time, $\mathrm{f}_{\mathrm{s}}=$ signal frequency.

\begin{tabular}{lcc}
\hline \hline Noise source & Max differential acceleration & Frequency content \\
\hline Brownian noise & $1 \times 10^{-14} \mathrm{~g} / \sqrt{\mathrm{Hz}}$ & white \\
Amplifier noise & $4 \times 10^{-15} \mathrm{~g} / \sqrt{\mathrm{Hz}}$ & white \\
Capsule's vibrations & $10^{-17} \mathrm{~g} / \sqrt{\mathrm{Hz}}$ & white \\
Drag in capsule & $6 \times 10^{-17} \mathrm{~g}$ & $1 / \mathrm{t}_{\mathrm{FALL}}$ \\
Proof-masses magnetic disturbances & $<10^{-17} \mathrm{~g}$ & $\mathrm{f}_{\mathrm{s}}$ \\
Radiometer effect & $2 \times 10^{-16} \mathrm{~g}$ & $\mathrm{f}_{\mathrm{s}}$ \\
Earth's gravity gradient torques & $10^{-16} \mathrm{~g}, 10^{-12} \mathrm{~g}$ & $\mathrm{f}_{\mathrm{s}}, 2 \mathrm{f}_{\mathrm{s}}$ \\
High-order gravitational coupling to capsule mass & $<10^{-16} \mathrm{~g}$ & $\mathrm{f}_{\mathrm{s}}, 2 \mathrm{f}_{\mathrm{s}}, 3 \mathrm{f}_{\mathrm{s}, \ldots}$ \\
Others & $<10^{-17} \mathrm{~g}$ & various \\
Error sum (rms), $\mathrm{t}_{\text {int }}=20 \mathrm{~s}$ & $2.4 \times 10^{-15} \mathrm{~g}$ & $\mathrm{f}_{\mathrm{s}}$ \\
\hline \hline
\end{tabular}




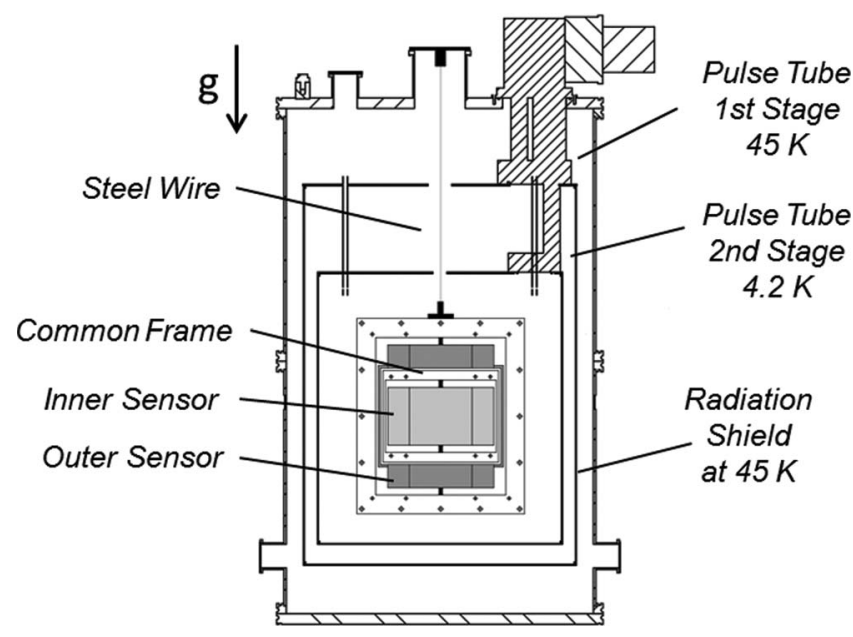

FIG. 5. Drawing of the experiment and of the cryogenic facility.

a cryostat using a pulse tube refrigerator and two cooling stages: a first stage at $45 \mathrm{~K}$ and a second stage at $4.2 \mathrm{~K}$. The pulse tube refrigerator is able to extract $40 \mathrm{~W}$ from the first stage and $1 \mathrm{~W}$ from the second stage.

In order to achieve the required low temperature, the sensor was shielded from thermal inputs associated with conduction and radiation. To this purpose, a second chamber was built within the main vacuum chamber to shield the sensor from thermal radiation by means of Multi-Layer Insulation sheets (MLI, silver Mylar).

\section{A. Single auto-differential accelerometer}

Measurements were carried out on the prototype differential accelerometer (previously described) that is aimed at testing and verifying key performance elements of the GReAT experiment.

At first, each single differential accelerometer was characterized to evaluate its behavior versus temperature. Then, both accelerometers were coupled and characterized as a unit (see Sec. III B). Figure 6 shows a detail of the experimental arrangement realized in the IAPS laboratory.

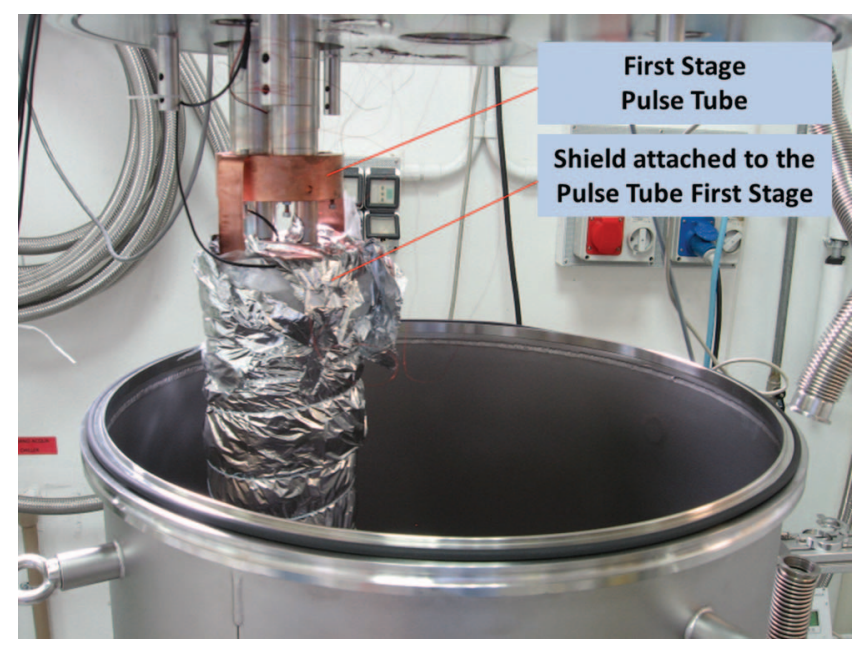

FIG. 6. Single implementation of the system showing the Mylar layers used to reduce the thermal input due to thermal radiation.
The sensor, complete of pick-up and actuator/control plates, was attached to the pulse tube refrigerator second stage by means of a mechanical flange and then to the vacuum chamber. A Si diode thermometer, able to measure temperatures lower than $1.4 \mathrm{~K}$, was placed on the accelerometer frame and monitored by a Lakeshore 336 controller. The sensor was interfaced with the electronics box, located outside the vacuum chamber, for signal acquisition and amplification. Finally, the temperatures were acquired through an ad hoc software expressly developed for these tests.

The preliminary characterization of the system was carried out sending pulses through the actuator/control plates, hence exciting oscillations of the sensor. Measurements were carried out on the system in vacuum (pressure $=10^{-4}$ mbar) at $293 \mathrm{~K}$.

It is well known that in a damped oscillator the amplitude decreases with time depending on the damping factor $1 / \tau$ and the amplitude ratio at two different times $t_{1}$ and $t_{2}$ is given by

$$
\frac{A\left(t_{1}\right)}{A\left(t_{2}\right)}=e^{-\Delta t / \tau},
$$

where $\mathrm{t}_{2}>\mathrm{t}_{1}$ and $\Delta \mathrm{t}=\mathrm{t}_{2}-\mathrm{t}_{1}$.

In order to obtain a correct measurement of the quality factor, the collected data sample was divided into time slots containing multiples of the oscillation period. For each time slot the PSD (Power Spectral Density) was evaluated to infer the amplitude peak of that sample at the resonance frequency.

The amplitude of the first period vs time was analyzed and the e-folding time $\tau$ was measured. On the basis of the value obtained, the $Q$ of the system was evaluated by the following equation:

$$
Q=\tau \pi v_{0}
$$

Figure 7 depicts the profile of the measured amplitudes (normalized) versus time (blue dots) and the exponential fit of the collected data. A value of $Q=57$ was measured in vacuum conditions with a pressure of $10^{-4}$ mbar at $293 \mathrm{~K}$.

Following this first measurement, the system was cooled down to $12 \mathrm{~K}$ monitoring the behavior of both resonance frequency and quality factor. Results are shown in Fig. 8.

The resonance frequency of the sensor increases with the decreasing temperature from $v_{0}=18.67 \mathrm{~Hz}$ at $293 \mathrm{~K}$ to

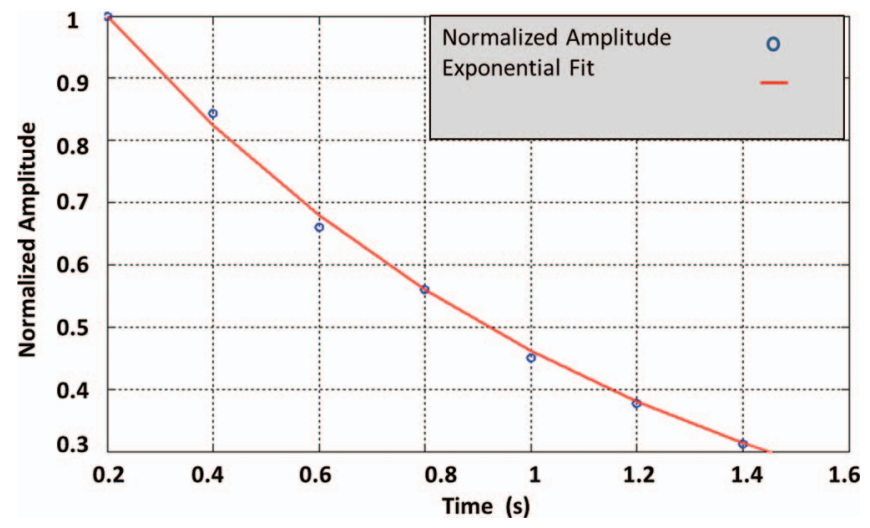

FIG. 7. Evaluation of the decay time of the signal to derive the quality factor (pressure is $10^{-4}$ mbar at $293 \mathrm{~K}$ ). 


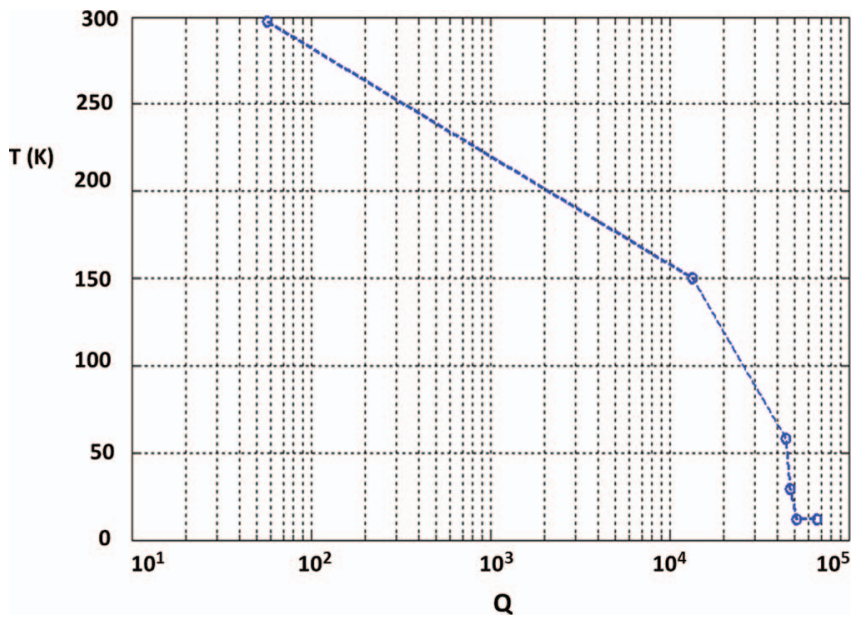

FIG. 8. Trend of the quality factor versus the temperature decreasing.

$v_{0}=19.71 \mathrm{~Hz}$ at $11.7 \mathrm{~K}$. At the same time the $\mathrm{Q}$ increases achieving a value of $7 \times 10^{4}$ at $11.7 \mathrm{~K}$. Data related to measurements carried out at $11.7 \mathrm{~K}$ are shown in Fig. 9.

From all the tests, it is possible to conclude that for low frequency harmonic oscillators $\left(v_{0}<20 \mathrm{~Hz}\right)$ the quality factor $\mathrm{Q}$ increases with decreasing temperature in a similar way as reported for high frequency oscillators (Borrielli et al. ${ }^{15}$ and Serra et al. ${ }^{16}$ ).

The value obtained $(Q \sim 70000)$ was still not high enough to match the scientific requirement. The authors hyphothesized that part of the mechanical energy was dissipated through the rigid fastening system. For this reason, the following tests were carried out uncoupling the vacuum chamber from the sensor. This objective was realized by suspending the experimental apparatus with a steel cable as discussed in what follows (see Fig. 5).

\section{B. Double auto-differential accelerometer}

The final tests were carried out on the system composed by two auto-differential accelerometers with common center of mass. The same measurements described in Sec. III A were carried out.

Since the GReAT experiment involves the free fall of the sensor, we approached the experimental tests isolating the

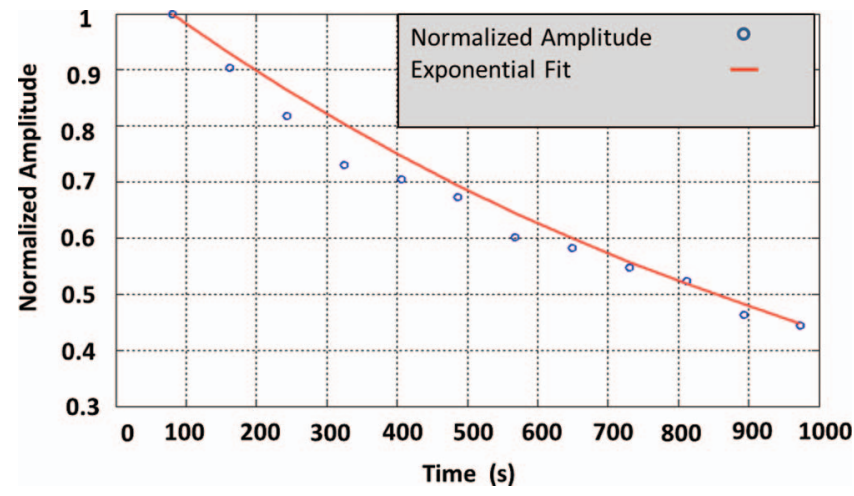

FIG. 9. Evaluation of the decay time of the signal to derive the quality factor (pressure is $10^{-4} \mathrm{mbar}$ at $11.7 \mathrm{~K}$ ).

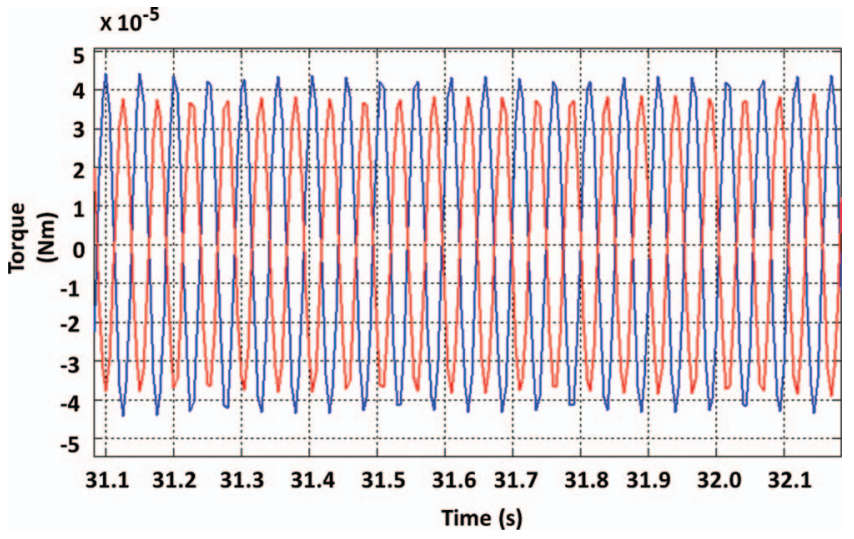

FIG. 10. Plot of single auto-differential accelerometers (red/smaller amplitude and blue) versus time for the differential mode, in vacuum at $11.7 \mathrm{~K}$.

sensor from any mechanical and thermal stress in order to mimic in the laboratory operational conditions in flight. Consequently, the sensor was suspended in the center of the vacuum chamber with a steel cable. This cable was placed coaxially with the torsional axis of the suspension blades in order to reduce vertical stresses.

This torsion balance-like configuration, thanks to its low torsional frequency, behaves as a filter for external disturbances.

However, the effectiveness of such a filter was reduced by the thermal links between the frame and the second stage of the cryogenic facility that increases the natural frequency of the torsion balance. The same links produce a loss of mechanical energy through the frame oscillations.

The coupling of two single oscillators produces a double oscillator exhibiting common-mode and differential mode oscillations. The common mode results from the in-phase movement of the proof masses, while the differential mode results from their phase-opposition motion. Such modes are resolved by monitoring the displacement of the proof mass of each single oscillator.

The common mode is excited through a stress imparted to the common frame of the oscillators, while the differential mode is excited by stressing each single proof mass. From the experimental point of view, such a stress was provided by the

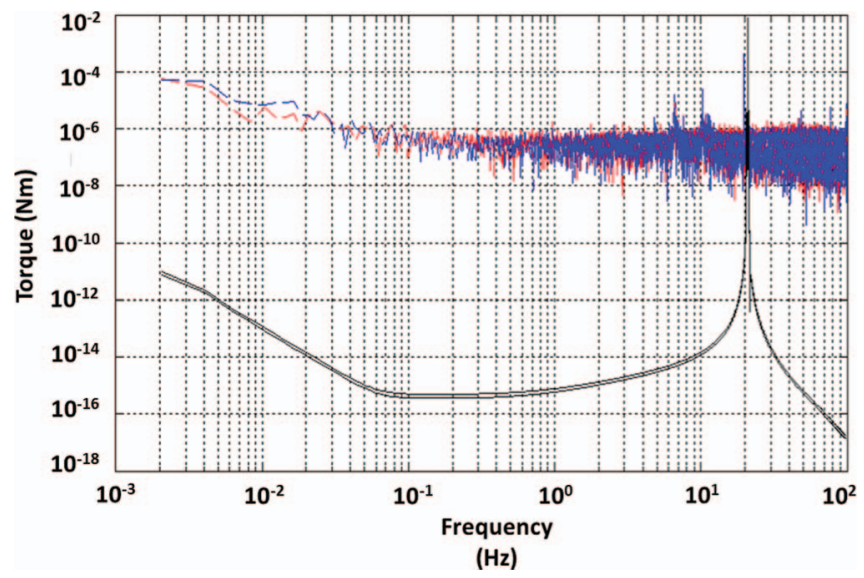

FIG. 11. PSD of the same signals as in the previous figure highlighting, through filtering, the differential mode at $19.6 \mathrm{~Hz}$. 


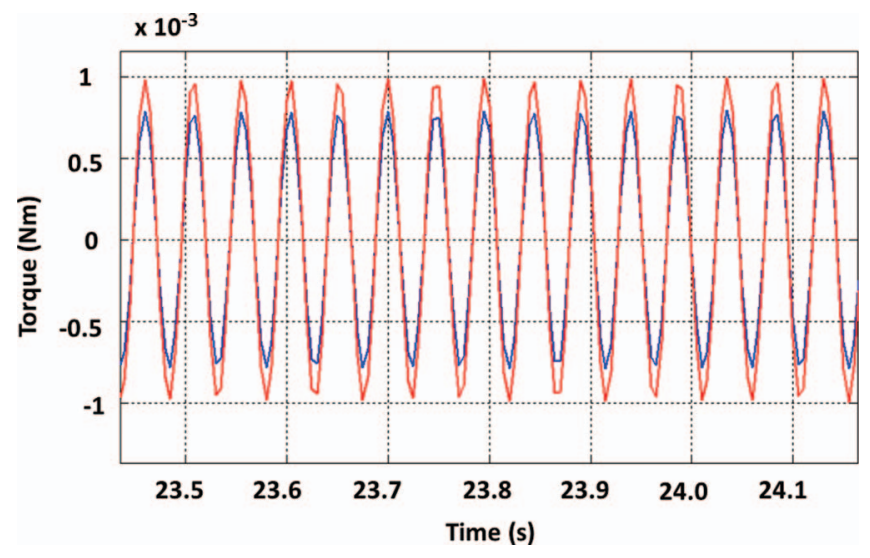

FIG. 12. Plot of single auto-differential accelerometers (red and blue/smaller amplitude) versus time for the common mode, in vacuum at $11.7 \mathrm{~K}$.

actuator/control plates. Note that in the free-fall experiment, any violation of the WEP would excite only the differential mode.

The system response, showing the free oscillations decay along with the associated PSD, is depicted in Figs. 10-13. Sensor outputs are shown in terms of torques by using the transduction factors of the two oscillators measured experimentally $\left(\alpha_{1}=0.17 \mathrm{~N} \mathrm{~m} / \mathrm{V}, \alpha_{2}=0.15 \mathrm{~N} \mathrm{~m} / \mathrm{V}\right)$.

The coupled system shows the combination of the single oscillator resonance frequencies stemming from the two normal modes, a differential mode at $19.6 \mathrm{~Hz}$ and a common mode at $20.9 \mathrm{~Hz}$.

As already mentioned, the filtered signals are in-phase or in phase-opposition depending on the mode type (common or differential).

The measurement of the quality factor was carried out by exciting the modes separately and evaluating the related decay time. Being orthogonal, the modes cannot be excited at the same time.

The common mode was excited through a mechanical perturbation applied to the frame. Figure 14 depicts the decay of common mode oscillations (normalized) versus time and a fit of the data by an exponential curve. The decay time is $\tau=668 \mathrm{~s}$, corresponding to a quality factor $Q=4.3 \times 10^{4}$.

For evaluating the differential mode decay, the actuator/control plates were utilized to excite the single proof mass.

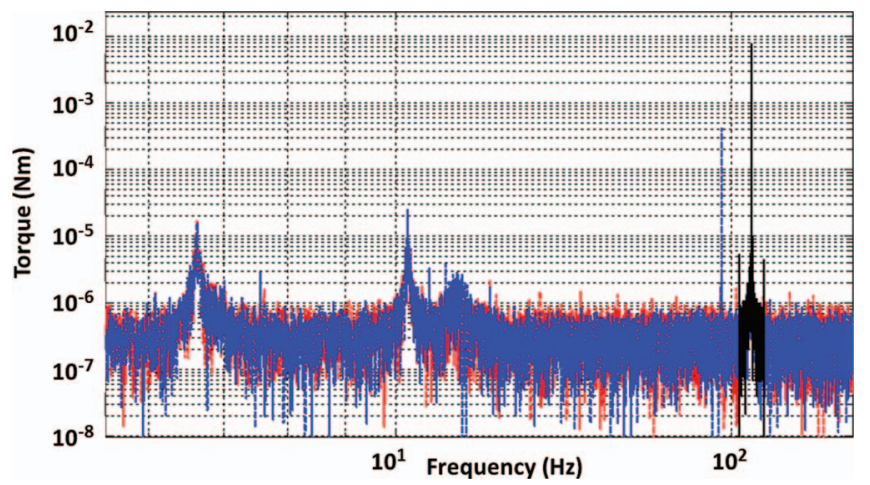

FIG. 13. PSD of the same signals highlighting, through filtering, the common mode at $20.9 \mathrm{~Hz}$.

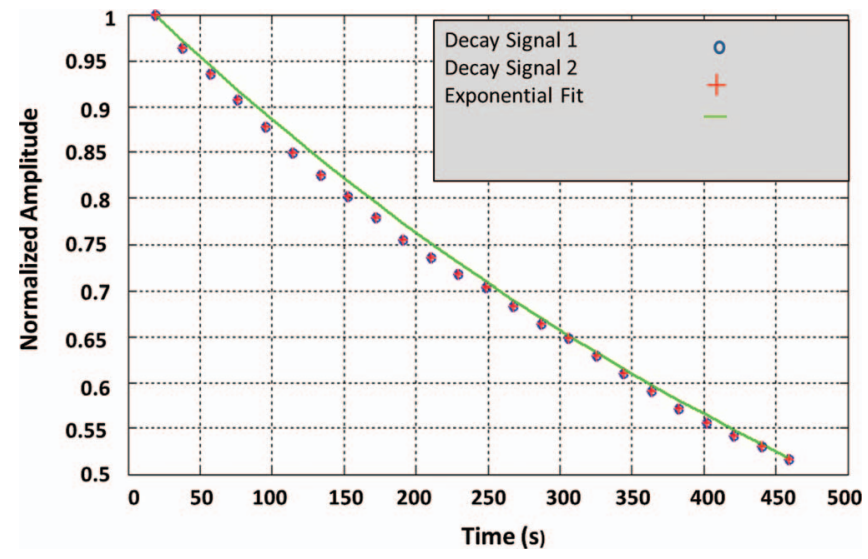

FIG. 14. Decay of the common-mode oscillations and related exponential fit.

A $480 \mathrm{~V}$ electric field at $1 \mathrm{kHz}$ was applied to the outer sensor in order to determine the coupling between the proof mass and the actuator plates.

The voltage was then modulated at the resonance frequency of the differential mode of $19.6 \mathrm{~Hz}$ to induce on the outer proof mass a (external) torque at the same resonance frequency, increasing the energy associated with that mode.

Figure 15 depicts the decay of the differential mode amplitudes for both output signals of the accelerometers. The figure depicts two zones: in Zone A there is an increase of the modulation frequency of the actuator/control plates; in Zone $\mathrm{B}$ the external torque is close to the resonance frequency of the differential mode and the energy acquired by the oscillations increases proportionally to $Q$.

The results of the $Q$ measurement for the differential mode are shown in Fig. 16.

The decay time was estimated to be $\tau=2000 \mathrm{~s}$, corresponding to a quality factor $Q=1.24 \times 10^{5}$.

The differential-mode quality factor is three times higher than the quality factor of the common-mode oscillation. This difference could be explained considering that the common mode includes an energy dissipation (not present in the differential mode) associated with the mechanical/thermal connection between the frame and the second stage of the pulse tube refrigerator.

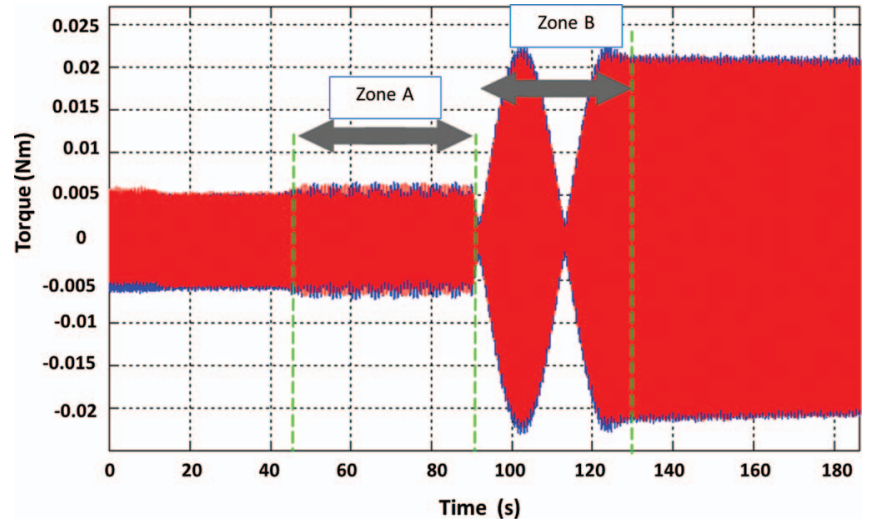

FIG. 15. Decay of the differential mode amplitude for both signals. 


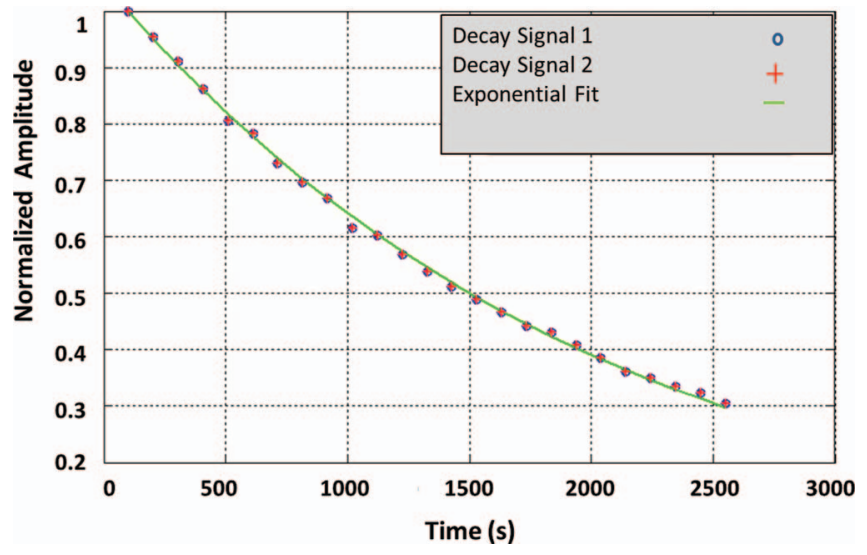

FIG. 16. Decay of the differential-mode oscillations and exponential fit.

\section{CONCLUSIONS}

The GReAT experiment is an experiment planned for testing the WEP with an improved accuracy $\left(\Delta a / a \approx 10^{-15}\right)$ with respect to the state of the art. The achievement of such a high accuracy is dependent on both the feasibility of a high performance sensor and the attainment of a high value of quality factor at cryogenic temperatures.

The experimental activity carried out at the EGG laboratory of IAPS allowed to design and manufacture a new differential accelerometer to test and verify key aspects of the sensor performance within the context of the GReAT experiment.

Specifically for this paper, tests were focused on verifying the behavior of the quality factor versus temperature in a low-resonant frequency sensor. Measurements proved that low frequency oscillators have a quality factor that increases as the temperature decreases. This behavior, experimentally tested, is consistent with the behavior observed in high-frequency oscillators. Concerning the quality factor of the sensor under testing, the authors achieved a $Q \sim 10^{5}$ for the differential mode by cooling the system at $\sim 12 \mathrm{~K}$. This value matches the accuracy requirement of the planned freefall experiment.

\section{ACKNOWLEDGMENTS}

This research work was supported by an Italian Space Agency (ASI) contract (ASI-INAF Agreement No. $\mathrm{I} / 014 / 09 / 0)$.

${ }^{1}$ V. Iafolla, E. Fiorenza, C. Lefevre, S. Nozzoli, R. Peron, M. Persichini, A. Reale, F. Santoli, E. C. Lorenzini, I. I. Shapiro, J. Ashenberg, C. Bombardelli, and S. Glashow, "GReAT (General Relativity Accuracy Test): A free fall test of weak equivalence principle from stratospheric balloon altitude," Memorie della SAIt (Società Astronomica Italiana) 79, 898
(2008); available online at http://sait.oat.ts.astro.it/MSAIt790308/PDF/ 2008MmSAI..79..898I.pdf.

${ }^{2}$ I. I. Shapiro, E. C. Lorenzini, J. Ashenberg, C. Bombardelli, P. N. Cheimets, V. Iafolla, D. M. Lucchesi, S. Nozzoli, F. Santoli, and S. Glashow, "Testing the principle of equivalence in an Einstein elevator," Int. J. Mod. Phys. D 16(12a), 2227-2243 (2007)

${ }^{3}$ E. C. Lorenzini, I. I. Shapiro, F. Fuligni, V. Iafolla, M. L. Cosmo, M. D. Grossi, P. N. Cheimets, and J. B. Zielinski, "Test of the weak-equivalence principle in an Einstein elevator," Il Nuovo Cimento B 109(11), 1195-1209 (1994).

${ }^{4}$ S. Schlamminger, K. Y. Choi, T. A. Wagner, J. H. Gundlach, and E. G. Adelberger, "Test of the equivalence principle using a rotating torsion balance," Phys. Rev. Lett. 100, 041101 (2008).

${ }^{5}$ J. G. Williams, S. G. Turyshev, and D. H. Boggs, "Progress in lunar laser ranging tests of relativistic gravity," Phys. Rev. Lett. 93, 261101 (2004).

${ }^{6}$ V. Iafolla, E. Fiorenza, C. Lefevre, D. M. Lucchesi, A. Morbidini, S. Nozzoli, R. Peron, M. Persichini, A. Reale, F. Santoli, E. C. Lorenzini, I. I. Shapiro, J. Ashenberg, C. Bombardelli, and S. Glashow, "TEPEE/GReAT (General Relativity Accuracy Test in an Einstein Elevator): Advances in the detector development," Il Nuovo Cimento C 031(4), 497-509 (2009).

${ }^{7}$ V. Iafolla, D. M. Lucchesi, S. Nozzoli, M. Ravenna, F. Santoli, I. I. Shapiro, E. C. Lorenzini, M. L. Cosmo, C. Bombardelli, J. Ashenberg, P. N. Cheimets, and S. Glashow, "General Relativity Accuracy Test (GReAT): New configuration for the differential accelerometer," Adv. Space Res. 47(7), 1225-1231 (2011).

${ }^{8}$ E. C. Lorenzini, I. I. Shapiro, J. Ashenberg, C. Bombardelli, P. N. Cheimets, V. Iafolla, D. M. Lucchesi, S. Nozzoli, F. Santoli, and S. Glashow, "Detector configurations for equivalence principle tests with strong separation of signal from noise," AIP Conf. Proc. 841, 502 (2006).

${ }^{9}$ C. M. Will, "The confrontation between general relativity and experiment," Living Rev. Relativity 9, 3 (2006).

${ }^{10}$ A. M. Nobili, G. L. Comandi, R. Pegna, D. Bramanti, S. Doravari, F. Maccarone, and D. M. Lucchesi, "Testing the weak equivalence principle," in Relativity in Fundamental Astronomy, Proceedings of the IAU Symposium No. 261, 2009 (International Astronomical Union, 2010).

${ }^{11}$ A. M. Nobili, "Precise gravitation measurements on Earth and in space: Tests of the equivalence principle," in Proceedings of the International School of Physics "Enrico Fermi," Course CXLVI, "Recent advances in metrology and fundamental constants" (Societa' Italiana di Fisica IOSS Press, 2001), pp. 609-645.

${ }^{12}$ G. Galilei, Discorsi e Dimostrazioni Matematiche intorno a due nuove scienze (Leiden, Louis Elsevier, 1638).

${ }^{13} \mathrm{~F}$. Fuligni and V. Iafolla, Galileo and the Principle of Equivalence (European Space Agency Publications, 1996), Vol. 115, pp. 104-112.

${ }^{14}$ D. Bramanti, G. Catastini, A. M. Nobili, E. Polacco, E. Rossi, and R. V. Caffarelli, Galileo and the Universality of Free Fall (ESA WPP, 1993), Vol. 115, p. 319.

${ }^{15}$ A. Borrielli, M. Bonaldi, and L. Conti, "Vibrational characterization of high-Q silicon resonators for investigating thermal noise statistical properties," in Proceedings of International Conference on Noise and Vibration Engineering (2012).

${ }^{16}$ E. Serra, M. Bonaldi, A. Borrielli, and L. Conti, "Vibrational characterization of high-Q silicon resonators for investigating thermal noise statistical properties," ISMA2012 - International Conference on Noise \& Vibration Engineering, Leuven, Belgium 17-19 September 2012, http://www.isma-isaac.be/isma_conf/prelimprogramme.html.

${ }^{17}$ R. G. Jackson, Novel Sensors and Sensing (IoP Publishing Ltd., 2004).

${ }^{18}$ M. A. Hopcroft et al., "Using the temperature dependence of resonator quality factor as a thermometer," Appl. Phys. Lett. 91, 013505 (2007).

${ }^{19}$ T. B. Gabrielson, "Mechanical-thermal noise in micromachined and acoustic and vibration sensors," IEEE Trans. Electron Devices 40(5), 903-909 (1993). 